\title{
Eficacia de biofungicidas frente a la caída de plántula de pepino, inducida por Pythium aphanidermatum
}

Efficacy of biofungicides against the fall of cucumber seedling, induced by Pythium aphanidermatum

\author{
Deissy Milly Leiva Villanueva ${ }^{1 *}$
}

\section{RESUMEN}

El objetivo de la presente investigación fue determinar la eficacia de ciertos tratamientos, a base de biofungicidas, frente a la caída de plántula de pepino causada por Pythium aphanidermatum. Los tratamientos a evaluar fueron 7 , incluyendo un tratamiento testigo, sin usar ningún producto. Los tratamientos usados fueron, un producto formulado con Bacillus subtilis, otro con Trichoderma fertile y otro formulado con Trichoderma asperellum, denominado T34 Biocontrol; como productos de referencia se utilizaron fungicidas químicos comerciales, en concreto Previcur Energy y Terrazole, y como biofungicida de control se utilizó Serenade Max. Se instaló un ensayo en cámara de cultivo con 5 bloques, 7 tratamientos y 4 repeticiones por tratamiento. Se pre-germinaron las semillas de pepino y posteriormente se sembraron en las macetas previamente identificadas. Seguidamente se inoculó a todos los tratamientos con un aislado de Pythium aphanidermatum.

Finalmente, se obtuvo que T34 Biocontrol logró reducir la incidencia de la enfermedad obteniendo el mismo resultado que el fungicida químico Previcur Energy. Así mismo, se demostró que el producto a base de Trichoderma fertile, también reduce la caída de plántula de pepino aunque no tan eficazmente como los otros tratamientos. Por último, los tratamientos con los biofungicidas Serenade Max y Bacillus subtilis no obtuvieron buenos resultados contra la caída de plántula en pepino. De igual manera, el fungicida quimico Terrazole tampoco tuvo buenos resultados contra la caída de plántula.

Palabras claves: Cucumis sativus, control biológico, fitopatogenos.

\begin{abstract}
The goal of the present research was to determine the efficacy of certain treatments, based on biofungicides, against the drop of cucumber seedling caused by Pythium aphanidermatum. The treatments to be evaluated were 7 , including a control treatment, without using any product. The treatments used were a product formulated with Bacillus subtilis, another with Trichoderma fertile and another formulated with Trichoderma asperellum, called T34 Biocontrol; as reference products, commercial chemical fungicides were used, specifically Previcur Energy and Terrazole, and Serenade Max was used as control biofungicide. An assay was installed in a culture chamber with 5 blocks, 7 treatments and 4 repetitions for treatment. The cucumber seeds were pre-germinated and subsequently planted in previously identified pots. All treatments were then inoculated with an isolate of Pythium aphanidermatum.

Finally, it was obtained that T34 Biocontrol managed to reduce the incidence of the disease obtaining the same result as the chemical fungicide Previcur Energy. Likewise, it so was demonstrated that the product based on Trichoderma fertile, also reduces the drop of cucumber seedling although not as efficiently as the other treatments. Finally, treatments with the biofungicides Serenade Max and Bacillus subtilis did not obtain good results against seedling fall in cucumber. Similarly, the chemical fungicide Terrazole also did not have good results against seedling fall.
\end{abstract}

Keywords: Cucumis sativus, biological control, phytopathogens.

\footnotetext{
Universidad de Sevilla, Calle San Fernando $N^{\circ} 4$, Sevilla, España
}

Autor de correspondencia. E-mail: deissy 51@hotmail.com 


\section{INTRODUCCIÓN}

Las plagas en los cultivos son una de las mayores causas en las perdidas económicas para los productores agrícolas, es por ello que dentro de las técnicas de control de las plagas se usan productos químicos y biológicos (como los biofungicidas) (Enríquez et al., 2010). En el control biológico de plagas, se usan agentes, los cuales son microorganismos que deben tener la capacidad de implantarse en la rizosfera y en la filosfera, creando una competencia entre ellos y el resto de microorganismo que están presentes en los cultivos (Zavaleta-Mejía, 1999; Casanova et al., 2008). Esto provoca que el efecto de las enfermedades producidas por patógenos sea mínimo, incluso en algunos casos activan mecanismos de defensa en las plantas. Los microrganismos usados en el control biológico suelen ser hongos como Clonastachys rosea o los hongos del género Trichoderma (Goñas et al., 2017). Sin embargo, es necesario que las medidas de control biológico que se implementen tienen que ser comprobadas científicamente y ser eficaces técnicamente (Lacey, 2015) La efectividad de los controles biológicos que se implementan se ve afectada por varios factores, como la climatología, la duración del control biológico, las características y propiedades edáficas o el contenido en humedad (Díaz-Hernández et al., 2017).

El peino (Cucumis sativus L.) es originario de la India, siendo domesticado en Asia y posteriormente introducido en Europa y América. Las variedades más comunes de pepino son el americano, el europeo, el holandés y el pepino oriental (Wehner y Maynard, 2003). Es por ello que es uno de los cultivos con más demanda mundial, al ser muy utilizado en la dieta del ser humano, ya que comerse un pepino equivale a beberse un vaso de agua, teniedo en cuenta que el $96 \%$ de su peso es agua y el $4 \%$ restante es materia solida con un gran valor nutritivo, pues es una gran fuente de minerales y vitaminas (Flores, 2017). El cultivo del pepino puede verse afectado por ciertos enemigos naturales, destacando sobre todo los hongos, las bacterias y los nematodos, afectando a la producción y productividad del cultivo (López-Elías, 2017).
Los hongos del género Pythium, son los responsables de que sea necesario abonar los cultivos en la agricultura trashumante (García, 2010), sin embargo su potencial como limitante de la productividad se confunde con otros factores por la compleja vida del suelo (Valdés-Rodríguez et al., 2011). Uno de los principales hongos fitopatógenos de este qénero es el Pythium aphanidermatum, un cromista del suelo con una amplia distribución global, muy común en regiones cálidas, ya que su crecimiento se ve favorecido por las temperaturas superiores a $30^{\circ} \mathrm{C}$. Normalmente, $P$. aphanidermatum suele dañar los frutos, semillas, raíces y bases del tallo en plántulas jóvenes (Parker, 2009). En concreto en el pepino, causa el ahogamiento de semillas y la caída de las plántulas (Sánchez y Gallego, 2002). Santos et al (2006), determinaron la capacidad supresora de un control bilógico como es el compost de orujo de vid sobre ciertos fitopatogenos que se dan en los cultivos de hortalizas, entre ellos $P$. aphanidermatum.

Es por ello que en la presente investigación se llevó a cabo la evaluación de la eficacia de tres biofungicidas: Un producto a formulado con Bacillus subtilis, otro con Trichoderma fertile y otro con Trichoderma asperellum (T34 Biocontrol); frente a los fungicidas químicos Terrazole y Previcur Energy y al biofungicida Serenade Max sobre caída de la plántula producida por Pythium aphanidermatum en pepino (Cucumis Sativa L.).

\section{MATERIALY MÉTODOS}

Se realizó un ensayo que consistió en evaluar la eficacia de biofungicidas, frente a la caída de plántula de pepino provocada por Pythium aphanidermatum, comparándolos con la eficacia de otros productos comerciales. El diseño fue de 5 bloques distribuidos completamente al azar, con 7 tratamientos y 4 repeticiones por tratamiento. Los biofungicidas de prueba utilizados en éste ensayo fueron:

- T34 BIOCONTROL: su materia activa es Tricoderma asperellum $10^{9} \mathrm{ufc} / \mathrm{g}, 10,83 \% \mathrm{p} / \mathrm{p}$.

- Producto a base de Bacillus subtilis: su materia

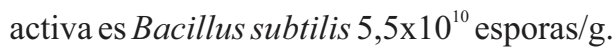


- Producto a base de Trichoderma fertile: su materia activa es Trichoderma fertile $2 \times 10^{9} \mathrm{ufc} / \mathrm{g}$.

Los productos comerciales de referencia fueron:

- PREVICUR ENERGY: fungicida cuyas materias activas son Fosetil 31\% p/v + Propamocarb 53\% $\mathrm{p} / \mathrm{v}$, se usa para aplicar al suelo y en pulverización foliar. Actúa por vía sistémica y contacto sobre las pudriciones de raíz-cuello producidas por Oomicetos de los géneros: Pythium y Phytophthora.

- TERRAZOLE: es un fungicida cuya materia activa es Etridiazol 48\%, de aplicación al suelo para el control de enfermedades de nascencia, de la raíz y de los tallos, causadas por hongos de los géneros: Pythium, Fusarium, Rhizoctonia y Phytophthora.

- SERENADE MAX: su materia activa es Bacillus subtilis 14,3\%. Características generales: previene y cura enfermedades foliares de la planta causada por hongos patógenos de las clases: Deuteromicetes, Oomicetes y Ascomicetes.

Se utilizó sustrato de turba (DSM1W KEKILA), especial para semillero hortícola. Su composición está basada en la mezcla de turba rubia. Posee una granulometría fina y $\mathrm{pH}$ corregido, con un agente humectante y fertilizante. Con la finalidad de homogenizar el sustrato, se humedecieron las bolsas y se dejaron incubando tres días antes de la instalación del ensayo. Estas bolsas contenían 12 litros de sustrato por bolsa, teniendo un total de 7 bolsas, una por tratamiento.

Se aisló el hongo $P$. aphanidermatum, a partir de plantas de pepino enfermas y posteriormente guardado en la Micoteca del Laboratorio de Sanidad Vegetal, E.T.S.I.A, Universidad de Sevilla. Para obtener cantidades suficientes de inóculo en cultivo, durante dos días el aislado de $P$. aphanidermatum se cultivó en placas con Agar jugo V8, para el cual se utilizaron los ingredientes indicados en la tabla 1.

Tabla 1. Ingredientes para la preparación del medio V8

\begin{tabular}{cc}
\hline Productos & Para 1 litro \\
\hline Zumo de vegetales (V8) & $200 \mathrm{ml}$ \\
Carbonato cálcico & $3 \mathrm{~g}$ \\
Agar & $20 \mathrm{~g}$ \\
Agua destilada & $800 \mathrm{ml}$ \\
\hline
\end{tabular}

Luego Se batió el agar de las placas con micelio con $100 \mathrm{ml}$ de agua estéril por placa con la batidora Ultramax Yellowline (Schott Ibérica S.A.) durante 3 minutos a 9500 r.p.m, obteniéndose finalmente una suspensión de micelio.

Para el ensayo se utilizaron semillas de pepino de la variedad 'Marketer' (Vilmorin, Saint-QuentinFallaber, Francia), variedad precoz y productiva, de frutos alargados conservándose verdes hasta bien desarrollados con piel lisa de color verde intenso. Se pusieron a pregerminar las semillas de pepino en cajas con papel de filtro húmedo durante tres días, previo al sembrado en las macetas. Posteriormente se llenaron 140 macetas con sustrato, para los 7 tratamientos, previamente identificados. En este ensayo se utilizaron macetas de 13 x $14 \mathrm{~cm}$ con un volumen de $600 \mathrm{ml}$. El mismo día se trasplantaron 10 plántulas por maceta, las plántulas al momento del trasplante presentaban dos cotiledones. Los tratamientos y dosis de los productos se pueden ver en la Tabla 2 .

Tabla 2. Dosis utilizadas según tratamientos

\begin{tabular}{ccc}
\hline Tratamientos & Producto & Dosis \\
\hline T1 & Testigo & 0 \\
T2 & Bacillus subtilis & $2,5 \mathrm{~g} / \mathrm{m}^{3}$ \\
T3 & Trichodermafertile & $0,25 \mathrm{~g} / \mathrm{m}^{3}$ \\
T4 & Serenade Max & $2,5 \mathrm{~g} / \mathrm{m}^{3}$ \\
T5 & PrevicurEnergy & $3 \mathrm{cc} / \mathrm{m}^{3}$ \\
T6 & Terrazole & $0,2 \mathrm{cc} / \mathrm{m}^{3}$ \\
T7 & T34 Biocontrol & $0,25 \mathrm{~g} / \mathrm{m}^{3}$ \\
\hline
\end{tabular}

Se hicieron dos inoculaciones del patógeno, la primera se aplicó a los ocho días y la segunda a los dieciséis a partir del pre germinado de semillas. Para todos los tratamientos, se aplicaron $25 \mathrm{ml}$ de inóculo por maceta en cada aplicación. El ensayo se realizó en una cámara de cultivo bajo un fotoperiodo de 12 horas luz y 12 horas oscuridad y una humedad relativa del $70 \%$ al $100 \%$ durante el transcurso del ensayo. El riego aplicado fue según el requerimiento del cultivo. Aproximadamente a los diez días del trasplante de plántulas, se empezó a medir enfermedad, cada lectura se realizó 3 veces por semana aproximadamente distinguiendo entre planta sana y planta afectada.

A los 30 días de la siembra se procedió al levantamien- 
to del ensayo, el mismo día se realizó una última lectura de enfermedad, así mismo se evaluó la incidencia final de la enfermedad, para ello se realizó el corte de plantas a nivel de cuello y se calculó por maceta el peso fresco de la planta y el peso seco de las plantas de pepino tras cuatro días en una estufa a $75^{\circ} \mathrm{C}$.

Se realizó un análisis factorial de la varianza para las variables de incidencia, peso fresco de la parte aérea de las plantas al final del ensayo y peso seco de la parte aérea de las plantas al final del ensayo. Para la separación de medias se empleó el test LSD $(\mathrm{P}<0,05)$. Para este análisis se usó el programa Statgraphics Plus versión 5.1.

\section{RESULTADOS Y DISCUSIÓN}

En la figura 1 se describieron los datos registrados de la incidencia de caída de plántula. A los 9 días después de la inoculación, los tratamientos no mostraron diferencias significativas.

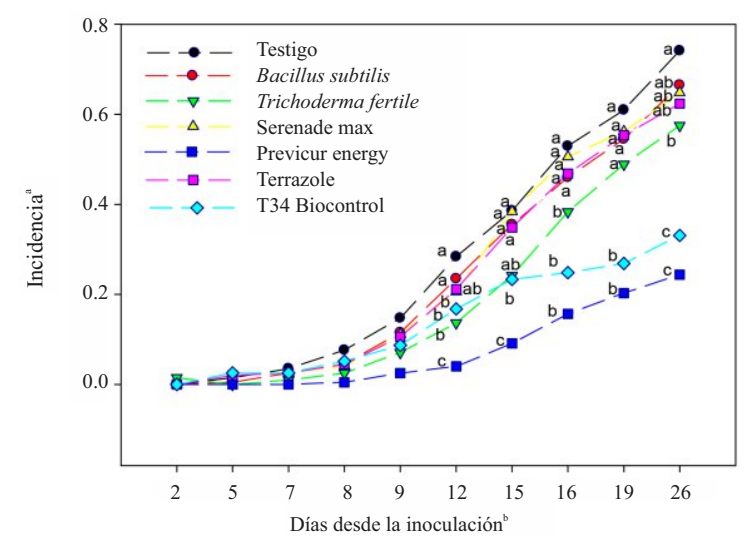

Figura 1. Evolución de la incidencia de la caída de plántula de pepino en los distintos tratamientos.

Todas las macetas fueron inoculadas con el patógeno Pythium aphanidermatum. Los valores son medias de los datos del ensayo. Las distintas letras pertenecen a grupos significativamente distintos. Estos grupos difieren $(\mathrm{P}<0,05)$, según el análisis de varianza seguido del test de LSD. Los datos para el análisis estadístico de los días: $12,15,16,19$ y 26 tras la inoculación fueron transformados con $\mathrm{x}^{0,2}, \mathrm{x}^{0,4}, \mathrm{x}^{0,5}, \mathrm{x}^{0,6} \mathrm{y} \mathrm{x}^{0,8}$ respectivamente.

b: Periodo de tiempo comprendido desde que la inoculación de macetas hasta el final del ensayo.

a: Incidencia media de la caída de plántula.

En el día 12 después de la inoculación, se comenzaron a observarse diferencias entre los distintos tratamientos, observándose como el testigo se desmarca mostrando más incidencias significativas en la caída de plántula que los tratamientos T34 Biocontrol, Tricho- derma fertile, Previcur Energy y Serenade Max.

Para el día 15 después de la inoculación, los tratamientos con Serenade Max, Terrazole, Bacillus subtilis y Trichoderma fertile son significativamente iguales al testigo; mientras que los tratamientos con T34 Biocontrol y Previcur Energy presentan significativamente una menor incidencia en comparación con el testigo. Sin embargo en el día 16 después de la inoculación, el tratamiento con Trichoderma fertile empieza a presentar una diferencia significativa frente al testigo, lo que quiere decir que este tratamiento empieza a frenar el avance de la caída de plántula al mismo nivel que los tratamientos con T34 Biocontrol y Previcur Energy.

El día 19 después de la inoculación, los tratamientos con Bacillus subtilis, Serenade max, Terrazole y Trichoderma fertile no muestran diferencias significativas con respecto al testigo; por lo que estos tratamientos no logran reducir la caída de plántula en el ensayo. Sin embargo los tratamientos con T34 biocontrol y Previcur Energy muestran menor incidencia.

Por último, que el día 26, correspondiente al final del ensayo, se puede apreciar como el testigo muestra una incidencia significativamente igual a los tratamientos con: Bacillus subtilis, Serenade Max y Terrazole. Sin embargo Trichoderma fertile es significativamente distinto al testigo, logrando reducir incidencia (Figura 1). Los tratamientos con Previcur Energy y T34 Biocontrol muestran una menor incidencia que el testigo y que Trichoderma fertile, por tanto son los mejores tratamientos obtenidos durante el ensayo. Por tanto el formulado de $T$. asperellum cepa T34 mantiene la eficacia contra la caída de plántula que se observó anteriormente (Casanova et al., 2008).

Khabbaz y Abbas (2014) demostraron que la bacteria Bacillus subtilis (aislado 8B-1) promueve el crecimiento vegetal y suprime la podredumbre de raíz y pudrición de plántulas de pepino en semilleros, causados por Pythium spp. Algo parecido observaron Virgen y García (1990), al obtener una reducción en la incidencia de Fusarium oxysporum f. sp. niveum, en plantas de sandía, que pertenece a la misma familia de los pepinos, mediante el tratamiento de semilla con Baci- 
llus subtilis, todo ello bajo condiciones de campo. Por otro lado, Virgen- Calleros et al. (2000) lograron determinar que ciertos fungicidas formulados con Bacillus subtilis tiener una gran eficacia en el control de Rhizoctonia solani en papa. Sin embargo en el ensayo realizado, los productos de Bacillus subtilis no han controlado caída de plántula en pepino.

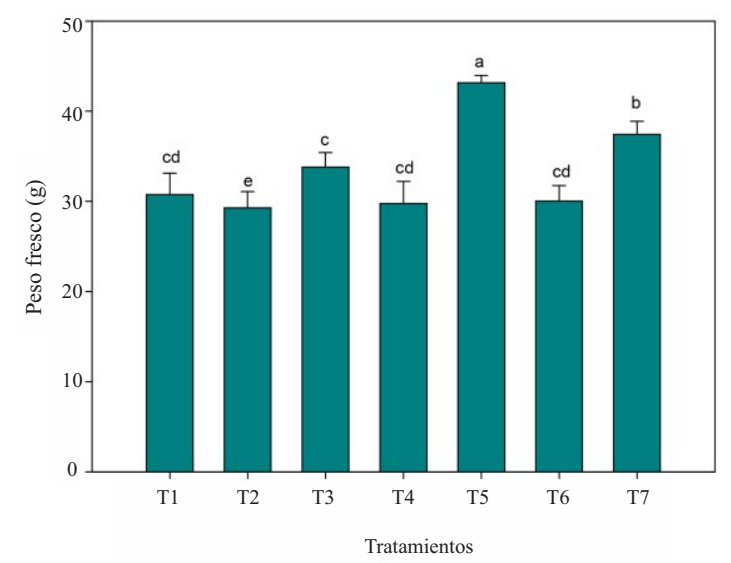

Figura 2. Peso freso de los diferentes tratamientos.

Todas las macetas fueron inoculadas con el patógeno Pythium aphanidermatum. Los valores son medias de los datos del ensayo. Las distintas letras pertenecen a grupos significativamente distintos. Estos grupos difieren $(\mathrm{P}<0,05)$, según el análisis de varianza seguido del test de LSD. Los datos para el análisis estadístico fueron transformados con $\mathrm{x}^{2,8}$.

En la figura 2, se puede ver que el peso fresco de las plantas correspondientes a los tratamientos con Previcur Energy, T34 Biocontrol es estadísticamente mayor que el testigo. Los tratamientos con Trichoderma fertile, Terrazole y Serenade Max son estadísticamente iguales comparados con el testigo; mientras que el tratamiento con B. subtilis presentó un menor peso fresco. Esto explica que Pythium aphanidermatum reduce el crecimiento vegetativo de las plantas y por tanto también el peso fresco total de cada maceta (Idowu et al., 2016). Así mismo esto refleja la incidencia de la enfermedad registrada durante el ensayo.

En la Figura 3, se observa que el peso seco del tratamiento con Previcur Energy difiere significativamente con el resto de tratamientos, esto corrobora lo dicho anteriormente y por tanto muestra que es el tratamiento más eficaz contra la caída la plántula. Esto mismo fue reportado por Scurtu et al. (2017) en guisantes (Pisum sativum), aplicando Previcur Energy en semillas, como protección ante ataques patógenos del suelo.

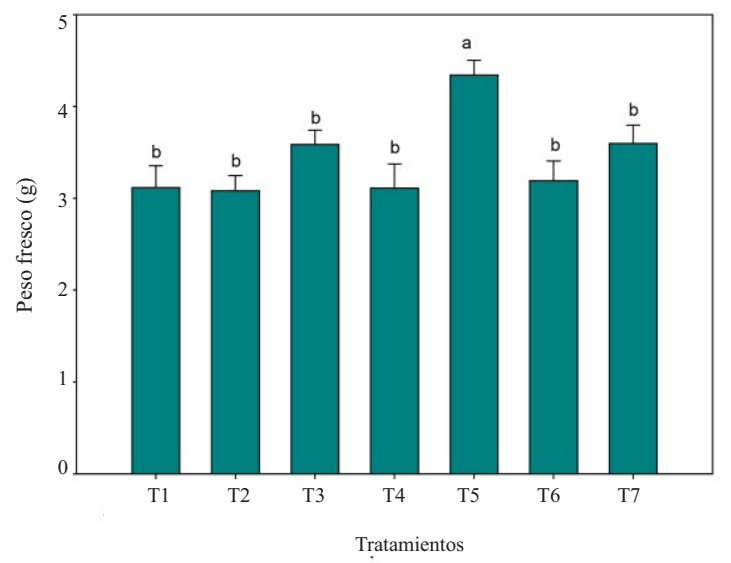

Figura 3. Peso seco de los diferentes tratamientos.

Todas las macetas fueron inoculadas con el patógeno Pythium aphanidermatum. Los valores son medias de los datos del ensayo. Las distintas letras pertenecen a grupos significativamente distintos. Estos grupos difieren $(\mathrm{P}<0,05)$, según el análisis de varianza seguido del test de LSD. Los datos para el análisis estadístico fueron transformados con $\mathrm{x}^{2}$.

\section{CONCLUSIONES}

Tras el ensayo podemos concluir que en las condiciones de ensayo estudiadas para la caída de plántula de pepino, el T34 Biocontrol, formulado con Trichoderma asperellum cepa T34, fue efectivo para el control de caída de plántula en pepino producida por Pythium aphanidermatum al mismo nivel que el tratamiento con el producto químico Previcur Energy.

En cuanto al biofungicida a base de Trichoderma fertile logró reducir caída de plántula en pepino, mientras que el biofungicida a base de Bacillus subtilis no fue efectivo para el control de caída de plántula en pepino producido por Pythium aphanidermatum.

Para finalizar, el biofungicida Serenade Max y el químico Terrazole, no lograron reducir la caída de plántula provocado por Pythium aphanidermatum en pepino.

\section{REFERENCIAS BIBLIOGRÁFICAS}

Casanova. E., P. Sánchez, G. Segarra, C. Borrero, M. Avilés, M. I. Trillas. 2008. "Beneficios del uso en la agricultura de agentes de control biológico Trichoderma asperellum cepa T34". En VIII Congreso SEAE de Agricultura y Alimentación Ecológica. Murcia (España).

Díaz-Hernández, S., L. Gallo-Llobet, P. DomínguezCorrea y A. Rodríguez. 2017. "Effect of 
repeated cycles of soil solarization and biosolarization on corky root, weeds and fruit yield in screen-house tomatoes under subtropical climate conditions in the Canary Islands". Soil Protection 94: 20-27.

Enríquez, M., M. Suquilanda, M. L. Tulcan. 2010. "Respuesta del pepinillo (Cucumis sativus L.) a tres métodos de desinfección de suelo". En XII Congreso Ecuatoriano de la Ciencia del Suelo. Tumbaco (Ecuador).

Flores, G. J. E. 2017. Análisis económico de la producción de pepino (Cucumis sativus L.) híbrido thunder, en el centro de prácticas Manglaralto provincia de Santa Elena. Tesis de Grado. Universidad Estatal Península de Santa Elena. La Libertad (Ecuador).

García, R. 2010. Agroecología y enfermedades de la raíz en cultivos agrícolas. México D.F. (México): Editorial BBA

Goñas, M., N. Y. Vera-Obando y S. T. Leiva. 2017. "Efecto antagónico in vitro de controladores biológicos sobre la pudrición gris de frutos de fresa (Fragaria ssp) en el distrito de Chachapoyas (Amazonas)". Revista de Investigación Agroproducción Sustentable 1 (1): 22-29.

Idowu, O. O., A. C. Oni y A. O. Salami. 2016. "The interactive effects of three Trichoderma species and damping-off causative pathogen Pythium aphanidermatum on emergence indices, infection incidence and growth performance of sweet pepper". International Journal of Recent Scientific Research 7 (4): 10339-10347

Khabbaz, S. E. y P. A. Abbasi. 2014. "Isolation, characterization, and formulation of antagonistic bacteria for the management of seedlings damping-off and root rot disease of cucumber". Canadian Journal of Microbiology 60 (1): 25-33.

Lacey, L. A., D. Grzywacz, D. I. Shapiro-Ilan, R. Frutos, M. Brownbridge y M. S. Goettel.
2015. "Insect pathogens as biological control agents: Back to the future". Journal of Invertebrate Pathology 132: 1-41.

López-Elías J., J. Jiménez, M. A. Huez, S. Garza, F. Cruz, A. L. Bautista. 2017. "Medidas de control biológico en la producción de pepino, bajo condiciones de invernadero". IDESIA 35 (3): 7-12.

Parker, K. C. 2009. Pythium aphanidermatum. Soilborne plant pathogens. Raleigh (EEUU): North Carolina State University.

Sánchez, J. y E. Gallego. 2002. "Fitopatogenicidad de Pythium spp. presentes en el agua de riego del Poniente almeriense (sureste de España)”. Revista Iberoamericana de Micología 19: 177-180.

Santos, M., F. Diánez, M. De Cara, J. C. Tello. 2006. "Supresividad del compost de orujo de vid frente a micosis edáficas de plántulas de hortalizas". Agroecología 1: 49-62.

Scurtu, I., G. Şovărel, A. E. Cenuşăa, M. Costache. 2017. "Protective effect of some fungicides used for the treatment of pea seed (pisum sativum L.)". Current Trends in Natural Sciences 6 (11): 58-62

Valdés-Rodríguez, O. A., R. García-Espinoza, O. Sánchez-Sánchez, A. Pérez-Vazquez. 2011. "Aislamiento y patogenicidad de un posible Pythium aphanidermatum en Jatropha Curcas L. no tóxica". Tropical and subtropical agroecosystems 14 (2): 649-660.

Virgen, G. y J. García. 1990. “Resultados preliminares sobre control biológico de Fusarium oxysporum f. sp. niveum con Bacillus subtilis en sandía, bajo condiciones de campo". En XVII Congreso Nacional de Fitopatología. Sinaloa (México).

Virgen-Calleros, G., V. Olalde-Portugal y D. E. Carling. 2000. "Anastomosis groups of Rhizoctonia solani on potato in Central Mexico and potential for biological and chemical control". American Journal of 
Potato Research 77:219-224.

Wehner, T. C. y D. N. Maynard. 2003. Cucumbers, melons, and other cucurbits. Volume 1. Encyclopedia of food and culture. Nueva York (EEUU): Editorial Scribner.

.Zavaleta-Mejía, E. 1999. “Alternativas de manejo de las enfermedades de las plantas". Terra Latinoamericana 17 (3): 201-207. 